\title{
Safe administration of bevacizumab combination chemotherapy for the patients with recurrent cervical cancer after pelvic radiotherapy: Two case reports
}

\author{
TADAHIRO SHOJI ${ }^{1}$, RYOSUKE TAKESHITA ${ }^{1}$, RIKA MUKAIDA $^{1}$, ERIKO TAKATORI ${ }^{2}$, \\ TAKAYUKI NAGASAWA ${ }^{2}$, HIDEO OMI ${ }^{2}$ and TORU SUGIYAMA ${ }^{2}$
}

${ }^{1}$ Department of Obstetrics and Gynecology, Hachinohe Red Cross Hospital, Hachinohe, Aomori 039-1104; ${ }^{2}$ Department of Obstetrics and Gynecology, Iwate Medical University School of Medicine, Morioka, Iwate 020-8505, Japan

Received December 27, 2017; Accepted May 24, 2018

DOI: $10.3892 / \mathrm{mco} .2018 .1642$

\begin{abstract}
In Japan, bevacizumab has not been proven either effective or safe for the treatment of recurrent cervical cancer. The present study reported two cases in which bevacizumab combination chemotherapy was safely administered for recurrent cervical cancer following pelvic radiotherapy. Case 1 was a 62-year-old woman with stage IIIB squamous cell carcinoma of the cervix who had received whole pelvic external beam radiotherapy (WPEBRT) at a dose of 50.4 Gy and high dose rate intra-cavitary brachytherapy at a dose of 24 Gy to the pelvis one year earlier. For recurrent cervical cancer, chemotherapy with paclitaxel, carboplatin and bevacizumab was administered for six cycles. Case 2 was a 52-year-old woman with stage IIB squamous cell carcinoma of the cervix who had received WPEBRT at a dose of 50.4 Gy to the pelvis 11 years earlier. For lymph node and liver metastases, chemotherapy with paclitaxel, cisplatin, and bevacizumab was administered for six cycles. Although grade 2 proteinuria was observed in one of these patients, there were no intestinal perforation, fistula, hypertension, proteinuria or thrombosis events, which are the characteristic adverse reactions associated with bevacizumab. Hematotoxicity was also manageable. Regarding the antitumor effect, case 1 demonstrated a complete response, whereas case 2 resulted in stable disease.
\end{abstract}

\section{Introduction}

Cisplatin has been used as monotherapy or in combination with other drugs as chemotherapy for treating cervical cancer $(1,2)$. Chemotherapy with taxanes has been studied in

Correspondence to: Dr Tadahiro Shoji, Department of Obstetrics and Gynecology, Hachinohe Red Cross Hospital, Nakaaketo 2, Tamonoki, Hachinohe, Aomori 039-1104, Japan

E-mail: tshoji@iwate-med.ac.jp

Key words: cervical cancer, bevacizumab, paclitaxel, cisplatin, carboplatin, radiotherapy recent years $(3,4)$. In the GOG204 study, which was a randomized controlled study involving 435 patients with stage-IVB or recurrent carcinoma of the uterine cervix, including 54 with adenocarcinoma and 36 with adenosquamous carcinoma, vinorelbine+cisplatin therapy, gemcitabine+cisplatin therapy, and topotecan+cisplatin therapy failed to show a therapeutic effect superior to that of paclitaxel+cisplatin therapy (5). Therefore, at present, combination chemotherapy with taxane plus a platinum agent is generally considered to be the most effective regimen for the treatment of cervical cancer. However, there is no established second-line chemotherapy for patients who have recurrent disease after receiving the first-line therapy with taxane plus a platinum agent. Bevacizumab, a recombinant humanized monoclonal antibody that limits angiogenesis by inhibiting vascular endothelial growth factor (VEGF) and the first molecular-target agent introduced in the gynecological field, has been established as a standard therapy for patients with cervical cancer based on the prolongation of PFS shown in previous studies. The Gynecologic Oncology Group (GOG) 240 trial demonstrated that bevacizumab combination chemotherapy improved the prognosis of advanced/recurrent cervical cancer (6). In Japan, after the tolerability of this chemotherapy regimen was demonstrated in a company-led clinical study on seven patients with advanced/recurrent cervical cancer (JO29569 study) (7), bevacizumab combination chemotherapy was approved for National Health Insurance reimbursement in May 2016. However, tolerability was assessed based on administration of one cycle in the Japanese study. The safety of bevacizumab combination chemotherapy for patients with recurrent cervical cancer after pelvic radiotherapy has still not been proven in Japan. We experienced two cases in which bevacizumab combination chemotherapy was safely administered for six cycles for recurrent cervical cancer after pelvic radiotherapy. These cases are described along with a literature review.

\section{Case reports}

Case 1. The patient was a 62-year-old woman with stage IIIB recurrent squamous cell carcinoma (SCC) of the cervix (gravida 3, para 3), with a history of cataract surgery at the age of 59 years and an unremarkable family history. 
At another hospital in May 2015, she received radiotherapy [whole pelvic external beam radiotherapy (WPEBRT) at a total dose of $50.4 \mathrm{~Gy}$ in 28 fractions and high dose rate intra-cavitary brachytherapy (HDR-ICBT) at a dose of 24 Gy per 4 fractions], which resulted in complete remission. She had subsequently been followed up. In May 2016, a recurrent tumor was detected in the uterine cervix, and she was referred to our hospital for treatment. At our department, SCC was detected by cervical biopsy. Magnetic resonance imaging (MRI) revealed a $2-\mathrm{cm}$ recurrent mass in the uterine cervix (Fig. 1A). Treatment consisted of paclitaxel at $175 \mathrm{mg} / \mathrm{m}^{2}$, carboplatin at an area under the curve of 6 , and bevacizumab at $15 \mathrm{mg} / \mathrm{kg}$. This regimen was administered every three weeks for six cycles. There was neither treatment delay nor dose modification. On MRI obtained at the end of the sixth cycle, the antitumor effect was determined to be complete response (CR) (Fig. 1B). Moreover, the serum SCC antigen level decreased from $2.5 \mathrm{ng} / \mathrm{ml}$ before treatment initiation to $1.2 \mathrm{ng} / \mathrm{ml}$ at the end of the sixth cycle. In addition cytological examination from the uterine cervix showed no evidence of disease. The observed adverse events are shown in Table I. During the two months since the end of treatment, no recurrence has been observed.

Case 2. A 52-year-old woman with stage IIB recurrent SCC of the cervix (unmarried), whose family and medical histories were unremarkable, underwent radical hysterectomy in June 2005. Because pelvic lymph node metastasis was detected, postoperative radiotherapy (WPEBRT at a total dose of 50.4 Gy in 28 fractions) was added. In 2010, metastatic lesions in the left lung were resected thoracoscopically. In 2011, she was diagnosed again as having left lung metastasis, for which left lower lobectomy and hilar/mediastinal lymphadenectomy were performed. After surgery, irinotecan (CPT-11) + etoposide therapy was added. In November 2012, metastasis recurred again in the left hilar region, and paclitaxel + carboplatin (TC) therapy was administered for six cycles, resulting in complete remission. In April 2013, she was diagnosed as having brain metastasis involving the left frontal lobe, for which whole brain radiotherapy (WBRT) was administered at a dose of 40 Gy in 20 fractions, resulting in complete remission. In August 2015, liver and para-aortic lymph node metastases were detected, and she was referred to our department for palliative treatment. However, because she strongly desired curative treatment, paclitaxel + cisplatin (TP) therapy was administered for nine cycles at our hospital. Subsequently, this therapy failed to induce remission and was switched to CPT-11 + cisplatin therapy. The administration of three cycles resulted in progressive disease (PD). Because she requested treatment with bevacizumab, the regimen was changed to paclitaxel at $135 \mathrm{mg} / \mathrm{m}^{2}$, cisplatin at $50 \mathrm{mg} / \mathrm{m}^{2}$, and bevacizumab at $15 \mathrm{mg} / \mathrm{kg}$. Six cycles were administered without treatment delay or dose modification. The computed tomography (CT) images taken before treatment initiation and at the end of the third and sixth cycles are shown in Fig. 2. At the end of the third cycle, the tumor growth rate was 5\%, and the antitumor effect was determined to be stable disease (SD). Although the chemotherapy was continued, the tumor growth rate was $37 \%$ at the end of the sixth cycle, and the antitumor effect was determined to be PD. Meanwhile, SCC grew from $1.9 \mathrm{ng} / \mathrm{ml}$ before treatment initiation to $1.5 \mathrm{ng} / \mathrm{ml}$ at the end of
Table I. Adverse events.

\begin{tabular}{lll}
\hline Adverse event & Case 1 & Case 2 \\
\hline Leucopenia & Grade 3 & Grade 3 \\
Neutropenia & Grade 4 & Grade 3 \\
Anemia & Grade 1 & Grade 3 \\
Thrombocytopenia & Grade 2 & Grade 1 \\
Nausea & Grade 1 & Grade 1 \\
Vomiting & Grade 0 & Grade 0 \\
Diarrhea & Grade 0 & Grade 0 \\
Fatigue & Grade 1 & Grade 1 \\
Sensory neuropathy & Grade 0 & Grade 2 \\
Myalgia & Grade 1 & Grade 2 \\
Mucositis & Grade 1 & Grade 0 \\
Hypertension & Grade 0 & Grade 0 \\
Proteinuria & Grade 2 & Grade 0 \\
GI perforation & Grade 0 & Grade 0 \\
Fistula & Grade 0 & Grade 0 \\
\hline
\end{tabular}

the third cycle and $3.1 \mathrm{ng} / \mathrm{ml}$ at the end of the sixth cycle. The observed adverse events are shown in Table I. Pegfilgrastim was prophylactically administered on day 3 of each cycle. As a result, febrile neutropenia did not occur, although grade 3 neutropenia did develop. Moreover, celecoxib had been orally administered for sensory neuropathy and myalgia. When both conditions progressed to grade 2, daily oral administration of goshajinkigan (TJ107) was initiated in the fifth cycle. At present, a change in the chemotherapeutic regimen is required and we are seeking an effective treatment alternative. Written informed consent was provided by the two patients prior to publication of the present case report.

\section{Discussion}

One of the serious adverse events caused by bevacizumab is gastrointestinal perforation (GIP). In clinical studies on ovarian cancer, namely GOG 218, the International Cooperative Group for Ovarian Neoplasia (ICON) 7, and Avastin Use in Platinum-Resistant Epithelial Ovarian Cancer (AURELIA) trials, the incidences of grade 2 or higher GIP were 1.7, 1.3 and $2.2 \%$, respectively (8-10). Meanwhile, the incidence in the GOG 240 trial was $2.3 \%$ (6). Because no evidence has been obtained, to date, proving that bevacizumab can be safely administered to Japanese patients with cervical cancer who have previously been treated with pelvic radiotherapy, we, Japanese gynecologists, hesitate to make the decision as to whether or not bevacizumab should be administered.

In our two cases, bevacizumab combination chemotherapy was initiated after we provided the patients with a sufficient explanation that the safety of bevacizumab therapy for cervical cancer has not been established in Japanese patients previously treated with radiotherapy and that the antitumor effect of bevacizumab combination chemotherapy has not been proven. For recurrent cervical cancer, TP therapy has been demonstrated to be effective (5). However, the Japan Clinical Oncology Group (JCOG) 505 trial proved that TC 

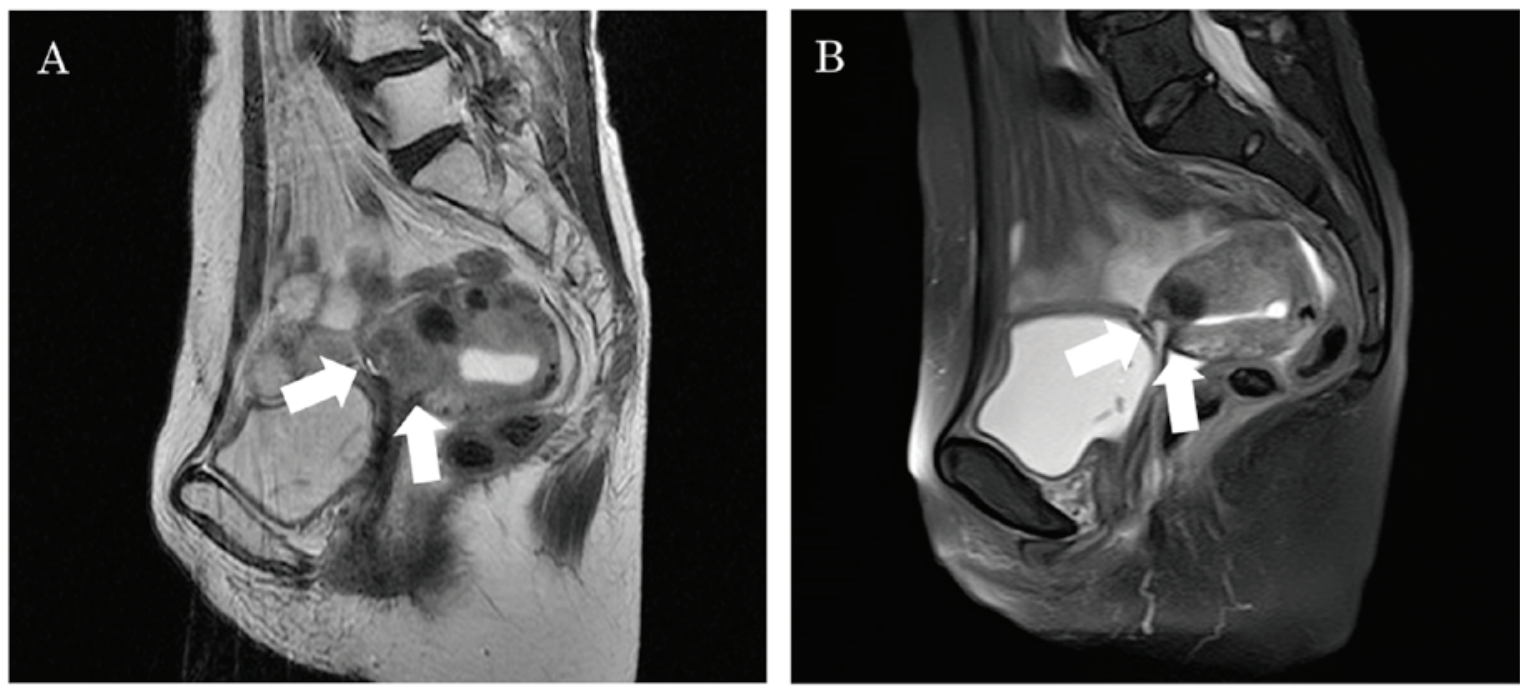

Figure 1. Antitumor effect observed by magnetic resonance imaging. The arrow shows a cervical recurrent mass. (A) T2-weighted magnetic resonance image obtained before treatment initiation. A $2 \mathrm{~cm}$ recurrent mass was detected in the uterine cervix. (B) T2-weighted fat saturated image obtained after the sixth cycle of chemotherapy. The recurrent mass in the uterine cervix has disappeared.

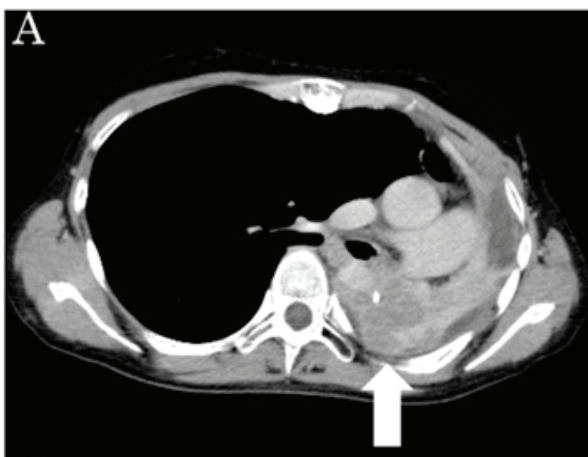

$\mathrm{D}$

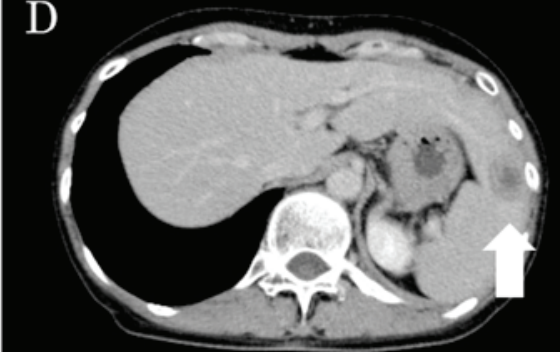

B

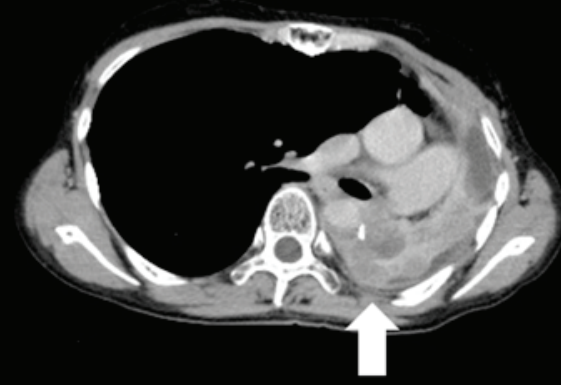

$\mathrm{E}$

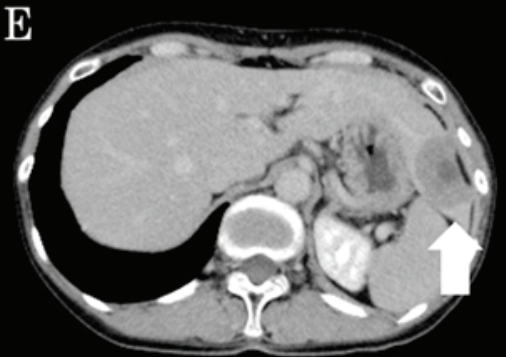

C

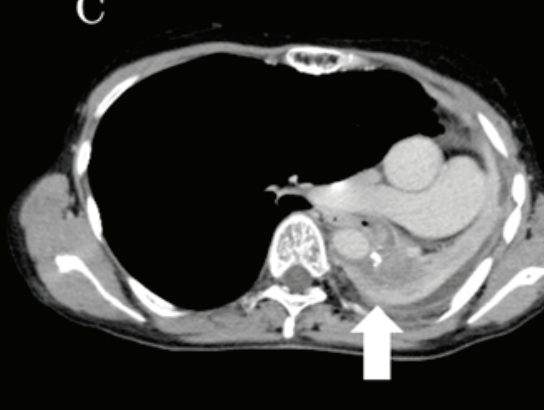

$\mathrm{F}$

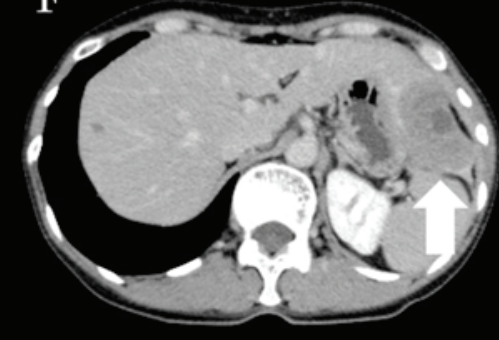

Figure 2. Antitumor effect observed by computed tomography. The arrow shows the para-aortic lymph node (A-C) and liver (D-F) metastases. (A) Contrast-enhanced chest computed tomography before treatment initiation. An irregularly demarcated mass shadow measuring $4 \mathrm{~cm}$ in the longest diameter was detected in the left posterior mediastinum. (B) Chest computed tomography after the third cycle of chemotherapy, the tumor was reduced to $3.3 \mathrm{~cm}$. (C) Chest computed tomography after the sixth cycle of chemotherapy, the tumor was increased to $3.7 \mathrm{~cm}$. The metastatic lesion in the left posterior mediastinum remained unchanged. (D) Contrast-enhanced abdominal computed tomography before treatment initiation. A metastatic lesion measuring $2 \mathrm{~cm}$ in the longest diameter was detected in segment 2 of the liver. (E) Abdominal computed tomography after the third cycle of chemotherapy, the tumor was increased to 3 cm. (F) Abdominal computed tomography after the sixth cycle of chemotherapy, the tumor was increased to $4.5 \mathrm{~cm}$. The metastatic lesion in segment 2 had grown. The tumor growth rate based on the Response Evaluation Criteria in Solid Tumors was 5\% at the end of the third cycle and $37 \%$ at the end of the sixth cycle.

therapy was non-inferior to TP therapy (11). Thus, in Case 1, TC therapy combined with bevacizumab was administered. Case 2 had been treated with five chemotherapeutic regimens, and each regimen had caused severe hematotoxicity. The utility of bevacizumab monotherapy for recurrent cervical cancer has not yet been proven. Therefore, for the treatment of the patients, we did not administer bevacizumab monotherapy, but instead administered a combination of paclitaxel, cisplatin and bevacizumab. We expected that another regimen would also cause severe hematotoxicity, cisplatin was selected instead of carboplatin. The dose of paclitaxel was reduced by one level to $135 \mathrm{mg} / \mathrm{m}^{3}(3 \mathrm{~h})$ in consideration of previous incidences of hematotoxicity and peripheral neuropathy due to chemotherapy. In Case 2, despite the occurrence of grade 3 neutropenia, febrile neutropenia did not develop due to prophylactic administration of pegfilgrastim. In Case 1, no granulocyte colony-stimulating factor preparations were administered. As for antiemetic drugs, palonosetron and dexamethasone were administered in 
Case 1 as described by Takatori et al (12), while a three-drug combination of aprepitant, palonosetron, and dexamethasone was administered in Case 2 according to the Multinational Association of Supportive Care in Cancer/European Society for Medical Oncology Antiemetic Guidelines (13). As a result, neither grade 2 or higher nausea nor vomiting was observed. In both cases, adverse events were manageable despite the administration of six cycles of this chemotherapy. Although bevacizumab was added, adequate supportive therapy, as described above, prevented serious adverse events and allowed six cycles of bevacizumab combination chemotherapy to be administered as safely as TC or TP therapy. Because there is as yet no evidence proving that continued administration of bevacizumab alone after six cycles is effective, we completed treatment with six cycles.

The most worrisome adverse events associated with bevacizumab treatment for recurrent cervical cancer are gastrointestinal and genitourinary fistulas. In the GOG240 trial, the incidence rates of grade 2 or higher gastrointestinal fistula, GIP, and genitourinary fistula were 5.0, 2.3, and 3.6\%, respectively. Despite their history of receiving radiotherapy, two patients in our study did not develop a fistula or GIP.

In Case 1, although she developed grade 2 proteinuria at the start of the second cycle, the chemotherapy was administered as planned because the urinary protein creatinine ratio was $£ 3.5$. The antitumor effect was determined to be CR in this patient. Although it was determined to be PD in Case 2 at the end of the sixth cycle, the best effect achieved was determined to be SD at the third cycle.

The radiotherapeutic procedure used in Japan is different from those used in Europe and the United States. In Japan, a midline block is used when 30 to 40 Gy of external beam radiation is administered, whereas no midline block is used in Europe or the United States. As for pelvic radiotherapy, HDR-ICBT is administered at a dose of $24 \mathrm{~Gy}$ in Japan, whereas low dose rate intra-cavitary brachytherapy (LDR-ICBT) is administered at a dose of 28 to 30 Gy in Europe and the United States $(14,15)$. Thus, although radiation doses to the intestinal tract are lower in Japanese than in Europeans and Americans, the incidence of GIP in Japan is not necessarily lower than $2.3 \%$, the rate observed in the GOG 240 trial, because HDR-ICBT is performed.

Furthermore, bevacizumab also reportedly increases the incidence of GIP in patients with ovarian cancer who have previously received three or more chemotherapy regimens (16). The safety of bevacizumb with respect to GIP has not been established for patients with a history of three or more chemotherapy regimens. Currently, there is no such concern over cervical cancer. In Case 2, although she had received four regimens, the bevacizumab combination chemotherapy was initiated after she had been confirmed to not meet any of the exclusion criteria for receiving bevacizumab therapy for ovarian cancer.

In our two cases, bevacizumab combination chemotherapy was administered for six cycles without causing GIP and fistula. This indicates that TP or TC therapy combined with bevacizumab may be an effective treatment strategy for cervical cancer. To verify that bevacizumab can be administered without causing GIP and fistula in Japanese patients with cervical cancer who have been treated with radiotherapy, phase II clinical studies need to be conducted. Although the Japanese Gynecologic Oncology Group (JGOG) has designed a clinical study to assess this issue, the study has not yet been started. The JO29569 study included only three patients who had previously been treated with pelvic radiotherapy (7). Although our report presents only two cases, it is the first description of Japanese patients with recurrent cervical cancer who had been treated with radiotherapy and then received six cycles of bevacizumab combination chemotherapy, with assessment for adverse events.

\section{Acknowledgements}

Not applicable.

\section{Funding}

No funding was received.

\section{Availability of data and materials}

The datasets used and/or analyzed during the current study are available from the corresponding author on reasonable request.

\section{Authors' contributions}

TSh and TSu conceived and designed this study. RT, RM, ET, TN and HO acquired the data and TSh, RT, and ET performed all the analysis. All authors read and approved the final manuscript.

\section{Ethics approval and consent for publication}

Not applicable.

\section{Consent for publication}

Written informed consent was provided by the two patients prior to publication of the present case report.

\section{Competing interests}

The authors declare that they have no competing interests.

\section{References}

1. Sugiyama T, Nishida T, Kumagai S, Nishino S, Fujivoshi K, Okura $\mathrm{N}$, et al: Combination chemotherapy with irinotecan and cisplatin as neoadjuvant in locally advanced cervical cancer. Br J Cancer 81: 95-98, 1999.

2. Tabata T, Nishiura K, Yanoh K, Okugawa T, Obata H, Tanaka K and Toyoda N: A pilot study of neoadjuvant chemotherapy with mitomycin $\mathrm{C}$, etoposide, cisplatin, and epirubicin for adenocarcinoma of the cervix. Int J Clin Oncol 9: 59-63, 2004.

3. Curtin JP, Blessing JA, Webster KD, Rose PG, Mayer AR, Fowler WC Jr, Malfetano JH and Alvarez RD: Paclitaxel, an active agent in nonsquamous carcinomas of the uterine cervix: A Gynecologic Oncology Group Study. J Clin Oncol 19: 1275-1278, 2001.

4. Shoji T, Kumagai S, Yoshizaki A, Yokoyama Y, Fujimoto T, Takano T, Yaegashi N, Nakahara K, Nishiyama $H$ and Sugiyama T: Efficacy of neoadjuvant chemotherapy followed by radical hysterectomy in locally advanced non-squamous carcinoma of the uterine cervix: A retrospective multicenter study of Tohoku Gynecologic Cancer Unit. Eur J Gynaecol Oncol 33: 353-357, 2012. 
5. Monk BJ, Sill MW, McMeekin DS, Cohn DE, Ramondetta LM, Boardman CH, Benda J and Cella D: Phase III trial of four cisplatin-containing doublet combinations in stage IVB, recurrent, or persistent cervical carcinoma: A Gynecologic Oncology Group study. J Clin Oncol 27: 4649-4655, 2009.

6. Tewari KS, Sill MW, Long HJ III, Penson RT, Huang H, Ramondetta LM, Landrum LM, Oaknin A, Reid TJ, Leitao MM et al: Improved survival with bevacizumab in advanced cervical cancer. N Engl J Med 370: 734-743, 2014.

7. Sugiyama T, Mizuno M, Aoki Y, Sakurai M, Nishikawa T, Ueda E, et al: A single-arm study evaluating bevacizumab, cisplatin, and paclitaxel followed by single-agent bevacizumab in Japanese patients with advanced cervical cancer. Jpn J Clin Oncol 46: 1-8, 2016

8. Burger RA, Brady MF, Bookman MA, Fleming GF, Monk BJ, Huang H, Mannel RS, Homesley HD, Fowler J, Greer BE, et al; Gynecologic Oncology Group: Incorporation of bevacizumab in the primary treatment of ovarian cancer. N Engl J Med 365 2473-2483, 2011

9. Perren TJ, Swart AM, Pfisterer J, Ledermann JA, PujadeLauraine E, Kristensen G, Carey MS, Beale P, Cervantes A, Kurzeder C, et al; ICON7 Investigators: A phase 3 trial of bevacizumab in ovarian cancer. N Engl J Med 365: 2484-2496, 2011.

10. Pujade-Lauraine E, Hilpert F, Weber B, Reuss A, Poveda A, Kristensen G, Sorio R, Vergote I, Witteveen P, Bamias A, et al: Bevacizumab combined with chemotherapy for platinum-resistant recurrent ovarian cancer: The AURELIA open-label randomized phase III trial. J Clin Oncol 32: 1302-1308, 2014

11. Kitagawa R, Katsumata N, Shibata T, Kamura T, Kasamatsu T, Nakanishi T, Nishimura S, Ushijima K, Takano M, Satoh T, et al Paclitaxel Plus Carboplatin Versus Paclitaxel Plus Cisplatin in Metastatic or Recurrent Cervical Cancer: The Open-Labe Randomized Phase III Trial JCOG0505. J Clin Oncol 33: 2129-2135, 2015
12. Takatori E, Shoji T, Miura Y, Nagao M, Takada A, Nagasawa T, Omi H, Kagabu M, Honda T and Sugiyama T: A phase II clinical trial of palonosetron for the management of delayed vomiting in gynecological cancer patients receiving paclitaxel/carboplatin therapy. Mol Clin Oncol 3: 281-286, 2015.

13. Dupuis LL, Roscoe JA, Olver I, Aapro M and Molassiotis A: 2016 updated MASCC/ESMO consensus recommendations: Anticipatory nausea and vomiting in children and adults receiving chemotherapy. Support Care Cancer 25: 317-321, 2017.

14. Nakano T, Kato S, Ohno T, Tsujii H, Sato S, Fukuhisa K and Arai T: Long-term results of high-dose rate intracavitary brachytherapy for squamous cell carcinoma of the uterine cervix Cancer 103: 92-101, 2005.

15. Erickson B, Eifel P, Moughan J, Rownd J, Iarocci T and Owen J: Patterns of brachytherapy practice for patients with carcinoma of the cervix (1996-1999): A patterns of care study. Int J Radiat Oncol Biol Phys 63: 1083-1092, 2005.

16. Cannistra SA, Matulonis UA, Penson RT, Hambleton J, Dupont J, Mackey H, Douglas J, Burger RA, Armstrong D, Wenham R, et al: Phase II study of bevacizumab in patients with platinum-resistant ovarian cancer or peritoneal serous cancer. J Clin Oncol 25 5180-5186, 2007.

This work is licensed under a Creative Commons Attribution-NonCommercial-NoDerivatives 4.0 International (CC BY-NC-ND 4.0) License. 